\title{
Structural Investigation of Iron(III) and Copper(II) Complexes with Poly(vinyl alcohol) by NMR Techniques
}

\author{
Yasuyoshi MorI, Hiroshi YokoI, ${ }^{\dagger}$ and Yutaka FusisE* \\ Department of Applied Chemistry, Faculty of Engineering, Shizuoka University, \\ 3-5-1 Johoku, Hamamatsu 432, Japan \\ * Department of Chemistry, Hamamatsu University School of Medicine, \\ 3600 Handa-cho, Hamamatsu 431-31, Japan
}

(Received August 25, 1994)

\begin{abstract}
Structures of the iron(III) and copper(II) complexes with poly(vinyl alcohol) (PVA) have been investigated in full detail by NMR techniques, especially through analysis of nuclear magnetic relaxation processes due to paramagnetic metal ions. As a result, it appears well established that the complexes are of a kind in which iron(III) or copper(II) hydroxide-like clusters are included by PVA chains with hydrophobic backbones toward the inside clusters and with hydrophilic $\mathrm{OH}$ groups toward the outside bulk water, meaning that the interaction between the clusters and PVA is of a hydrophobic type. By such an interaction, the complexes have certain definite structures, because there is always a linear relationship between the ${ }^{1} \mathrm{H}$ NMR signal intensity of PVA and the concentration of metal ion in every system of complex formation. In the case of the PVA of not less than 250 in the degree of polymerization, however, there has also appeared another linear relationship with a more gentle slope over a relatively high concentration range of iron(III), suggesting that such PVA molecules can form both complexes containing only one and two or more clusters.
\end{abstract}

KEY WORDS Poly(vinyl alcohol) / Degree of Polymerization / Iron(III) Complex / Copper(II) Complex / Metal Hydroxide-Like Cluster / ${ }^{1} \mathrm{H}$ NMR Signal Intensity / NMR Relaxation Rate / Hydrophobic Interaction /

Poly(vinyl alcohol) (PVA) is a most simple synthetic water-soluble polymer bearing only alcoholic $\mathrm{OH}$ groups. It is quite interesting that PVA has a property of forming complexes with inorganic ions and compounds and even organic substances in a wide $\mathrm{pH}$ range ${ }^{1-6}$; some of the complexes are colloids or gels. Much attention has been paid to the usefulness of these complexes as functional materials. ${ }^{1,7}$

Iron(III) and copper(II) ions in aqueous solution hydrolyze and precipitate as hydroxides at $\mathrm{pH}>3$ and $>6$ respectively, whereas, in the presence of PVA, they form red brown and green water-soluble complexes respectively. ${ }^{8-10} \mathrm{Up}$ to recently, it has been believed that these complexes are of a type in which each metal ion directly coordinates to the deprotonated or intact $\mathrm{OH}$ groups of PVA. ${ }^{11}$ However, we have shown by magnetic susceptibility measurements and transmission electron microscopic examination that these metal ions exist as metal hydroxide-like clusters with a diameter of about $\sim 30 \AA .^{8-10}$ From this result, it has been reasonably concluded that metal ions do not coordinate to the $\mathrm{OH}$ groups of PVA and the interaction between PVA and the clusters are of a hydrophobic type. Furthermore, experiments on the dependence of the degree of polymerization (DP) of.PVA on the complex formation have revealed that the cluster was composed of at most 120 metal ions per PVA chain. ${ }^{10}$ However, little is known

\footnotetext{
† To whom all correspondence should be addressed.
} 
of structure of these complexes.

The purpose of this paper is to investigate the structures of iron(III) and copper(II) complexes with PVA extensively by utilizing NMR techniques thoroughly. Both iron(III) and copper(II) ions are paramagnetic, and nuclear magnetic relaxation processes due to these ions become inevitably important here. Many PVAs of different degrees of polymerization were prepared and used for elucidating DP effects on the structure of the complexes.

\section{EXPERIMENTAL}

\section{Synthesis of PVA}

The polymerization of vinyl acetate (Tokyo Kasei, reagent grade) was initiated by $\alpha, \alpha^{\prime}$-azobisisobutyronitrile (AIBN) (Tokyo Kasei, reagent grade) in $i$-propanol (Nakarai tasque, reagent grade) at $70^{\circ} \mathrm{C}$. The solvent was selected because of its fairly large chain transfer constant. The DP of poly(vinyl acetate) (PVAc) was controlled by varying the quantity of the solvent, ${ }^{12}$ and determined by viscosity measurements in acetone at $30^{\circ} \mathrm{C} .{ }^{13}$ The PVAc was saponified with $\mathrm{NaOH}$ in methanol at room temperature. The degree of saponification (DS) was controlled by varying the quantity of the methanol solvent. ${ }^{12}$ The PVA isolated was washed with methanol in a Soxhlet's extractor and dried in vacuo at $30^{\circ} \mathrm{C}$ for 2 or 3 days. The seven PVAs of different DP-DS values prepared here are as follows: 180-100, 130-98, 130-91, 77-97, 77-92, 33-95, and 33-90. The DS was determined by the NMR method, within an error of $\pm 1 \%$. Beside these PVAs, commercially-available PVAs (Unitika, 250-98; Unitika, 400-99; Fluka, 500-100) were also used.

\section{Preparation of the Aqueous Solutions of Metal Complexes with PVA}

In this study, the concentration of PVA is expressed in terms of the monomeric residue, and $R=$ [PVA monomeric residue]/[metal ion] is used as a parameter for a specification of the solutions. Sample solutions were pre- pared by mixing $\mathrm{D}_{2} \mathrm{O}$ (Matheson, 99.9 atom \% D) solutions of $\mathrm{Fe}\left(\mathrm{ClO}_{4}\right)_{3} \cdot 6 \mathrm{H}_{2} \mathrm{O}$ (Kanto Chemicals, reagent grade) or $\mathrm{Cu}\left(\mathrm{ClO}_{4}\right)_{2} \cdot 6 \mathrm{H}_{2} \mathrm{O}$ (Kanto Chemicals, reagent grade) and PVA at desired $R$ values, and carefully adjusted to desired $\mathrm{pD}$ values with $\mathrm{NaOD}$ without any buffer, where $\mathrm{pD}$ was determined by adding the factor of 0.4 to the usual $\mathrm{pH}$-meter reading. ${ }^{14}$ Poly(vinyl methyl ether) (PVME) (Tokyo Kasei, 30\% in water), a PVA derivative of methyl ether, was also used in comparison with PVA in the complex formation with iron(III).

\section{Measurements}

${ }^{1} \mathrm{H}$ NMR spectra were recorded at $25 \pm$ $0.1^{\circ} \mathrm{C}$ with JEOL GSX-270 and EX-90 NMR spectrometers at 270 and $90 \mathrm{MHz}$ respectively. All ${ }^{1} \mathrm{H}$ NMR signal intensities were expressed in values relative to that of 3-(trimethylsilyl)propionic-2,2,3,3,- $d_{4}$ acid, sodium salt (TSP) (Aldrich, 99 atom \% D) as an external standard. Longitudinal relaxation times $\left(T_{1}\right)$ for ${ }^{1} \mathrm{H}$ NMR signals were determined by the inversion-recovery technique. ${ }^{15}$ In this process, at least 8 repetition times were selected properly enough for the determination of every $T_{1}$ value. Transverse relaxation time $\left(T_{2}\right)$ were determined by the CPMG method. ${ }^{16}$ These relaxation times were reproducible to about $\pm 2 \%$ on repeated runs. Iron(III) and copper(II) ions are paramagnetic. The observed relaxation rate, $\left(1 / T_{1}\right)_{\text {obs }}$, is

$$
\left(\frac{1}{T_{1}}\right)_{\mathrm{obs}}=\left(\frac{1}{T_{1}}\right)_{\mathrm{p}}+\left(\frac{1}{T_{1}}\right)_{0}
$$

where two terms on the right side are the relaxation rate due directly to the paramagnetic ions and the one in the absence of those ions respectively. ${ }^{1} \mathrm{H}$ or ${ }^{13} \mathrm{C}$ relaxation rates in solutions containing paramagnetic ions are quantitatively related to the correlation time, $\tau_{c}$, and the distance, $r$, between the magnetic nuclei and metal ion in the complexes, as expressed in the Solomon-Bloembergen equa- 
tion. ${ }^{17}$ If the electron spin relaxation time of metal ion is of the order of $10^{-10} \mathrm{~s}$, as is the case with high-spin iron(III) ion, $\tau_{c}$ is dependent upon the NMR radio frequency. In this study, we used the Bloembergen-Morgan equation $^{18}$ developed for such a case and obtained eq 2 by calculating the ratio of two $\left(1 / T_{1}\right)_{p}$ values measured at two different NMR frequencies $\left(\omega_{1}, \omega_{2}\right) .{ }^{19}$

$$
\frac{\left(\frac{1}{T_{1 \mathrm{p}}}\right)_{\omega_{1}}}{\left(\frac{1}{T_{1 \mathrm{p}}}\right)_{\omega_{2}}}=\frac{1+\omega_{2}{ }^{2}\left(\frac{\omega_{2}}{\omega_{1}}\right)^{4} \tau_{\mathrm{c}}{ }^{2}}{\left(\frac{\omega_{2}}{\omega_{1}}\right)^{2}\left(1+\omega_{1}{ }^{2} \tau_{\mathrm{c}}{ }^{2}\right)}
$$

Properly speaking, eq 2 must be applied only to these complexes which have fixed coordination structures of their own, such as hydrated metal ions. Although the coordination structure of the present complexes with PVA is unknown owing to the formation of metal hydroxide-like clusters, eq 2 has been used here in order to estimate $\tau_{\mathrm{c}}$ for convenience. As a result, some interesting information on the structure of the complexes has been obtained for the first time, as will be described below.

\section{RESULTS AND DISCUSSION}

Concentration Dependence of Metal Ions on the ${ }^{1} \mathrm{H} N M R$ Intensity of PVA

Figure 1 shows the ${ }^{1} \mathrm{H}$ NMR spectra of iron(III) complex with PVA under the conditions of [PVA monomeric residue]= $100 \mathrm{mM}$ and $\mathrm{pD}$ 7.0. Marked line-broadening and reduction of signal intensity are observed for all the signals of methine, methylene, and acetate methyl groups with increasing concentration of iron(III). This signal reduction was determined quantitatively, as shown in Figures 2 and 3 , where the concentration dependence of iron(III) and copper(II) ions on ${ }^{1} \mathrm{H}$ NMR intensities of PVA respectively can be clearly seen. Protons in close proximity of paramagnetic iron(III) and copper(II) ions apparently exhibit no detectable NMR signals owing

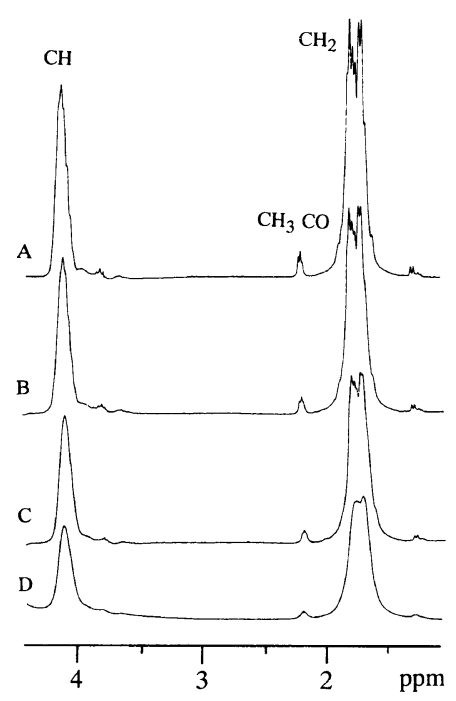

Figure 1. ${ }^{1} \mathrm{H}$ NMR spectra $(270 \mathrm{MHz})$ of PVA-iron(III) complex $(\mathrm{DP}=250 ; \mathrm{DS}=98$; [PVA] $=100 \mathrm{~mm} ; \mathrm{pD} 7.0$; $\left.25 \pm 0.1^{\circ} \mathrm{C}\right): \mathrm{A}$, PVA alone; $\mathrm{B}, R=50 ; \mathrm{C}, R=25 ; \mathrm{D}, R=15$.

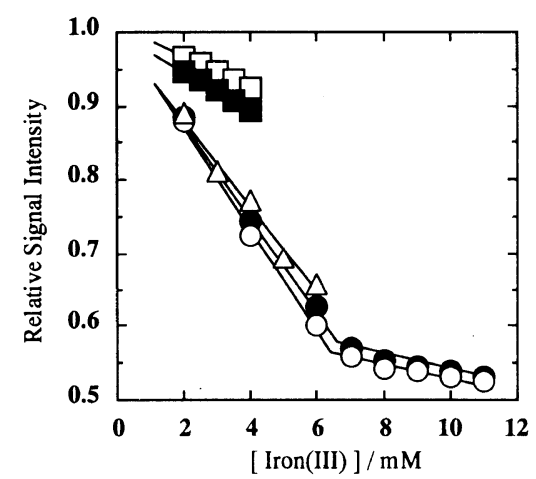

Figure 2. Plots of the relative ${ }^{1} \mathrm{H}$ NMR signal intensity of the $\mathrm{CH}_{2}$ group of PVA against the concentration of iron(III) by the use of various PVAs of different DP values $\left([\mathrm{PVA}]=100 \mathrm{mM} ;\right.$ pD $\left.7.0 ; 25 \pm 0.1^{\circ} \mathrm{C}\right): \quad \mathrm{O}, \mathrm{DP}=400$ $(\mathrm{DS}=100) ; \bigcirc, \mathrm{DP}=250(\mathrm{DS}=98) ; \triangle, \mathrm{DP}=180(\mathrm{DS}=$ $100) ; \square, \mathrm{DP}=130(\mathrm{DS}=98) ; \square, \mathrm{DP}=77(\mathrm{DS}=97)$.

to remarkable line broadening, and hence their signal intensities apparently reduce. The amount of such protons can be estimated from the degree of reduction in their signal intensities. In Figures 2 and 3, the relationship between the relative signal intensity and the concentration of metal ions is linear in every case, although the slope is somewhat dependent upon the DP. The linearity itself demonstrates 


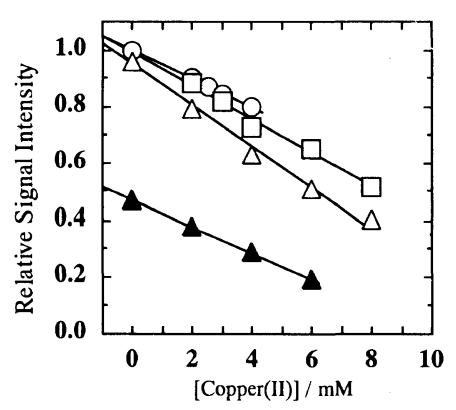

Figure 3. Plots of the relative ${ }^{1} \mathrm{H}$ NMR signal intensity of PVA against the concentraion of copper(II) by the use of various PVAs of different DP values ([PVA] $=100 \mathrm{mM}$; $\left.25 \pm 0.1^{\circ} \mathrm{C} ; \mathrm{pD} 8.0\right): \mathrm{O}, \mathrm{CH}_{2}(\mathrm{DP}=33 ; \mathrm{DS}=95) ; \square, \mathrm{CH}_{2}$ $(\mathrm{DP}=250 ; \mathrm{DS}=98) ; \triangle, \mathrm{CH}_{2}(\mathrm{DP}=400 ; \mathrm{DS}=100) ; \Delta, \mathrm{CH}$ $(\mathrm{DP}=400 ; \mathrm{DS}=100)$.

that every complex formed in these concentration ranges of PVA and metal ions has a certain definite structure and, therefore, a definite interaction between PVA and metal ions, where metal ions are in the form of metal hydroxide-like clusters. PVA monomeric residues which are in close proximity of metal clusters and, in other words, directly surround the clusters have no detectable ${ }^{1} \mathrm{H}$ NMR signals. The number of such PVA monomeric residues per iron(III) $\left(N_{\mathrm{VA} / \mathrm{M}}\right)$ in each case can be calculated from the slope of line in Figures 2 and 3. In each case with iron(III) and the PVA of DP $\geqq 250$, however, two different lines in relatively high and low concentration ranges of iron(III) respectively were observed, as shown in Figure 2. This fact indicates that there are two different types of interaction between PVA and iron(III). All the $N_{\mathrm{VA} / \mathrm{M}}$ values obtained here are summarized in Table I.

In both iron(III) and copper(II) systems, $N_{\mathrm{VA} / \mathrm{M}}$ increases with the DP of PVA, and levels off around DP $=500$, as shown in Table I. In the former system, however, there is a gap in $N_{\mathrm{VA} / \mathrm{M}}$ between the DPs of 130 and 180, suggesting that the interaction between PVA and iron(III) is different in type on the higher and lower sides of the gap. Similarly, there was a gap between the DPs of 130 and 180 in the ability to prevent the iron(III)-hydroxide
Table I. The number of ${ }^{1} \mathrm{H}$ NMR signal-disappearing PVA monomeric residues per metal ion $\left(N_{\mathrm{VA} / \mathrm{M}}\right)^{\mathrm{a}}$

\begin{tabular}{ccc}
\hline System & DP of PVA & $N_{\text {VA/M }}$ \\
\hline PVA-Fe(III) & 77 & 2.0 \\
& 130 & 2.5 \\
& 180 & 5.8 \\
& 250 & $6.1^{\mathrm{b}} ; 1.2^{\mathrm{c}}$ \\
& 400 & $6.2^{\mathrm{b}} ; 1.2^{\mathrm{c}}$ \\
PVA-Cu(II) & 500 & $6.2^{\mathrm{b}} ; 1.2^{\mathrm{c}}$ \\
& 33 & 5.1 \\
& 250 & 6.0 \\
& 400 & 7.4 \\
& 500 & $7.2^{\mathrm{d}}$ \\
\hline
\end{tabular}

a $25 \pm 0.1^{\circ} \mathrm{C} ;[\mathrm{PVA}]=100 \mathrm{mM} ; \mathrm{DS} \simeq 100 .{ }^{\mathrm{b}}$ In the range of $[\operatorname{Iron}(\mathrm{III})] \lesssim 7.5 \mathrm{mM}$. ${ }^{\mathrm{c}}$ In the range of $[\operatorname{Iron}(\mathrm{III})] \gtrsim$ $7.5 \mathrm{~mm}$. ${ }^{\mathrm{d}}$ Reference 9.

precipitation. ${ }^{10}$ An explanation for this phenomenon is that, on the higher side, a PVA molecule interacts with an iron(III) cluster, while, on the lower side, two or more PVA molecules collaboratively do. On the other hand, it is also interesting that there are two different types of interaction between the PVA of DP $\geqq 250$ and iron(III) in relatively high and low concentration ranges of iron(III) respectively. The $N_{\mathrm{VA} / \mathrm{M}}$ values in the former range are much smaller than those in the latter, and, furthermore, smaller than those in the case of the PVA of DP $\leqq 130$. An possible explanation for this small $N_{\mathrm{VA} / \mathrm{M}}$ value is that, in a relatively high concentration range of iron(III), a PVA molecule of DP $\geqq 250$ may form a complex with an assembly of two or more iron(III) clusters. This is supported by the experimental result of $\mu_{\text {eff }}=3.62$ and 3.69 B.M. for the complexes with the PVA of DP $=250$ in the high and low concentration ranges of iron(III) respectively. Both $\mu_{\text {eff }}$ values are almost equal and in agreement with the results reported previously. ${ }^{8,10}$ This fact, together with the fact of $\mu_{\text {eff }}=4.6$ B.M. for the iron(III) hydroxide precipitate produced at $\mathrm{pH} \sim 7,{ }^{20}$ suggests that the complexes formed in both concentration ranges of iron(III) include the same kind of iron(III) clusters. It is also suggested that the 
same kind of definite iron(III) clusters are always formed by the hydrolysis of iron(III) ions in the presence of PVA, as if it plays such a catalytic role as some proteins do in biomineralization. ${ }^{21}$ It is to be noted that the two types of complex formation are characteristic of the PVA of DP $\geqq 250$.

Iron(III) and copper(II) have the coordination numbers of 6 and 4 respectively, and produce structurally different hydroxides or oxides. $^{22}$ This fact seems to reflect the difference in $N_{\mathrm{VA} / \mathrm{M}}$ between the iron(III) and copper(II) complexes with PVA, as shown in Table I.

\section{Paramagnetic Ion-Induced ${ }^{1} H$ NMR Relaxa- tion}

Figures $4 \mathrm{~A}$ and $4 \mathrm{~B}$ show the $\mathrm{pD}$ dependence of the paramagnetically induced spin-lattice relaxation rate $\left(1 / T_{1 \mathrm{p}}\right)$ of PVA protons in the aqueous solutions of iron(III) and copper(II) complexes respectively. Figures $4 \mathrm{~A}$ demonstrates that the values of $1 / T_{1 \mathrm{p}}$ for the $\mathrm{CH}_{2}$ and $\mathrm{CH}$ groups of PVA remarkably decrease up to $\mathrm{pD} 4$ and are kept constant at $\mathrm{pD}>4$. Since the iron(III) clusters formed remain unchanged in structure at $\mathrm{pH}>4$ and each complex has a certain definite structure, as mentioned above, the interaction between PVA and the iron(III) cluster must be in a definite and uniform way. This is the reason why both values of $1 / T_{1 \mathrm{p}}$ for the $\mathrm{CH}_{2}$ and $\mathrm{CH}$ groups are constant at $\mathrm{pD}>4$. Interestingly, the former $1 / T_{1 p}$ value is larger than the latter, as shown in Figure 4A. This fact indicates that the $\mathrm{CH}_{2}$ protons are situated in positions closer to the iron(III) cluster surface on the average than $\mathrm{CH}$ ones. The same experimental result has also been obtained for the system of PVA and copper(II), as shown in Figure 4B. The periphery of the $\mathrm{CH}_{2}$ groups is more hydrophobic in nature than that of the $\mathrm{CH}$ groups to which the hydrophilic $\mathrm{OH}$ group is bonded. The above-mentioned experimental finding that the $\mathrm{CH}_{2}$ groups are favorably closer to the iron(III) or copper(II) cluster
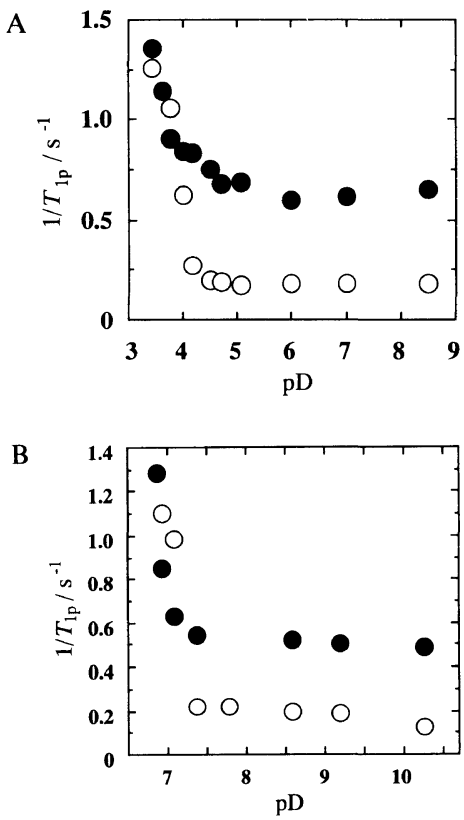

Figure 4. $\mathrm{pD}$ dependence of the paramagnetic contribution of iron(III) (A) and copper(II) (B) to $1 / T_{1}$ for the ${ }^{1} \mathrm{H}$ NMR $(90 \mathrm{MHz})$ of PVA (DP $=250, \mathrm{DS}=98$; [PVA] $=$ $\left.400 \mathrm{~mm} ; R=40 ; 25 \pm 0.1^{\circ} \mathrm{C}\right): \mathrm{CH}_{2} ; \mathrm{O}, \mathrm{CH}$.

surface than the $\mathrm{CH}$ groups, being accompanied by the result that the $\mathrm{OH}$ groups are relatively apart from the cluster surface, suggests that the interaction between PVA and the iron(III) or copper(II) cluster may be of a hydrophobic type, as has already been proposed by us. ${ }^{8,9}$ This is also supported by the fact that the $\mathrm{CH}_{2}$ group is more dependent in the ${ }^{1} \mathrm{H}$ NMR signal intensity upon the concentration of metal ion than the $\mathrm{CH}$ group, as shown in Figure 3. On the other hand, at $\mathrm{pD}<4$, there is no difference in $1 / T_{1 \mathrm{p}}$ between the $\mathrm{CH}_{2}$ and $\mathrm{CH}$ groups of PVA, as shown in Figures 4A and 4B. This may be interpreted as indicating that the $\mathrm{CH}_{2}$ and $\mathrm{CH}$ groups are equivalent in the paramagnetic enhancement of ${ }^{1} \mathrm{H}$ NMR relaxation time, because the complex formation is incomplete in this $\mathrm{pD}$ range.

According to the Solomon-Bloembergen equation, the difference between $1 / T_{1 \mathrm{p}}$ and $1 / T_{2 p}$ increases with an increasing relative 
Table II. $1 / T_{1 \mathrm{p}}$ and $1 / T_{2 \mathrm{p}}$ for the aqueous solutions of PVA and iron (III) or copper(II) ${ }^{a}$

\begin{tabular}{ccccccc}
\hline \multirow{2}{*}{ System } & & \multicolumn{2}{c}{$1 / T_{1 \mathrm{p}} / \mathrm{s}^{-1}$} & & \multicolumn{2}{c}{$1 / T_{2 \mathrm{p}} / \mathrm{s}^{-1}$} \\
& & $\mathrm{CH}$ & $\mathrm{CH}_{2}$ & & $\mathrm{CH}$ & $\mathrm{CH}_{2}$ \\
\hline PVA $^{\mathrm{b}}-\mathrm{Fe}(\mathrm{III})$ & 3.5 & 1.25 & 1.32 & & 1.36 & 1.92 \\
& 7.0 & 0.23 & 0.64 & & 0.27 & 0.76 \\
PVA $^{\mathrm{b}}-\mathrm{Cu}(\mathrm{II})$ & 6.8 & 2.23 & 1.41 & & 3.58 & 3.07 \\
& 7.4 & 0.17 & 0.60 & & 0.19 & 0.60 \\
\hline
\end{tabular}

${ }^{\mathrm{a}}[\mathrm{PVA}]=400 \mathrm{mM} ; R=40 ; 25 \pm 0.1^{\circ} \mathrm{C}$; measured at 90 $\mathrm{MHz} .{ }^{b} \mathrm{DP}=130 ; \mathrm{DS}=98$.

contribution of the scalar relaxation, ${ }^{23}$ as exemplified by the ${ }^{13} \mathrm{C}$ NMR relaxation of a copper(II) complex with polyglutamic acid, where $1 / T_{2 p}$ is larger than $1 / T_{1 \mathrm{p}}$ in the ${ }^{13} \mathrm{C}$ NMR signals, because a significant amount of electron spin density is transferred to $\mathrm{C}_{\gamma}$ from the copper(II) ion coordinated to a carboxylic group. ${ }^{24}$ The experimental results of $1 / T_{1 \mathrm{p}}$ and $1 / T_{2 \mathrm{p}}$ for ${ }^{1} \mathrm{H}$ NMR signals of iron(III) and copper(II) complexes with PVA are summarized in Table II. Both relaxation rates are comparable in magnitude for all ${ }^{1} \mathrm{H}$ NMR signals at any $\mathrm{pH}$ value, suggesting that the ${ }^{1} \mathrm{H}$ NMR relaxation enhanced by paramagnetic ions in these complexes comes dominantly from the dipolar interaction but little from both scalar interaction and chemical shift anisotropy and, hence, that direct coordination of the paramagnetic ions to the hydroxyl groups of PVA in the complexes seems quite unlikely.

In order to obtain further conformational information on the hydroxyl groups of PVA in their complexes, $1 / T_{1 \mathrm{p}}$ was measured for all ${ }^{1} \mathrm{H}$ NMR signals in the complexes with the PVA of a low DS and PVME; the results are summarized in Table III. Both $\mathrm{CH}_{2}$ and $\mathrm{CH}$ protons of PVA are comparable in $1 / T_{1 \mathrm{p}}$ independently of DS and the kind of metal ion, as well as $\mathrm{CH}_{2}$ protons of PVA and PVME. Roughly speaking, this table indicates that all the complexes with the PVA of any DS and with PVME have common structural features,
Table III. $1 / T_{1 \mathrm{p}}$ for the aqueous solutions of PVA and iron(III) or copper(II) and of PVME and Iron (III) ${ }^{\mathrm{a}}$

\begin{tabular}{ccccc}
\hline \multirow{2}{*}{ System } & & \multicolumn{4}{c}{$1 / T_{1 \mathrm{p}} / \mathrm{s}^{-1}$} \\
\cline { 3 - 5 } & & $\mathrm{CH}_{2}$ & $\mathrm{CH}_{3}{ }^{\mathrm{b}}$ & $\mathrm{CH}$ \\
\hline PVA-iron(III) $^{\mathrm{c}}$ & 91 & 1.57 & 0.78 & 1.42 \\
& 98 & 1.75 & & 1.69 \\
PVA-copper(II) $^{\mathrm{d}}$ & 91 & 1.43 & 0.35 & 1.22 \\
& 98 & 1.45 & & 1.21 \\
PVME-iron(III) $^{\mathrm{e}}$ & & 1.46 & 0.71 & - $^{\mathrm{f}}$ \\
\hline
\end{tabular}

${ }^{\mathrm{a}}[\mathrm{PVA}]=[\mathrm{PVME}]=400 \mathrm{mM} ; \mathrm{PVA}, \mathrm{DP}=130, \mathrm{DS}=$ $98 ; R=40 ; 25 \pm 0.1^{\circ} \mathrm{C}$; measured at $90 \mathrm{MHz}$. ${ }^{\mathrm{b}}$ in acetyl (PVA) or methyl ether (PVME) group. ${ }^{\mathrm{c}}$ Measured at $\mathrm{pD}$ 4.0. ${ }^{\mathrm{d}}$ Measured at $\mathrm{pD} 7.0 .{ }^{\mathrm{e}}$ Measured at $\mathrm{pD} 3.5 .{ }^{\mathrm{f}}$ Not observed owing to signal overlapping.

regardless of the kind of metal ion. The methyl protons of PVA with a low DS and of PVME are smaller in $1 / T_{1 p}$ than the $\mathrm{CH}_{2}$ ones in the polymer backbone. This indicates that the methyl groups are apart from the metal ions than the $\mathrm{CH}_{2}$ ones. In conclusion, it is suggested that the $\mathrm{OH}$ groups of PVA in the complexes may be oriented toward the outside bulk water and, hence, that the interaction between PVA and the metal ion clusters is of a hydrophobic type.

\section{${ }^{13}$ C NMR Relaxation and Tacticity of PVA in the Complex Formation}

The methine carbon atom resonances of PVA appear as three distinct features assigned to isotactic, heterotactic, and syndiotactic triad sequences in order of increasing field. ${ }^{25}$ In order to obtain information on effects of the tacticity of PVA on the structures of the complexes, $1 / T_{1 \mathrm{p}}$ was measured for these ${ }^{13} \mathrm{C}$ NMR signals. The results are summarized in Table IV. There are no remarkable difference in $1 / T_{1 \mathrm{p}}$ among the three triad sequences at any $\mathrm{pD}$. Therefore, it seems probable that the tacticity of PVA has no influence on the complexation. It is well known that the property of PVA in aqueous solution depends on its tacticity, ${ }^{26}$ especially because of a marked effect of the tacticity on the formation 
Table IV. $1 / T_{1 \mathrm{p}}$ for the ${ }^{13} \mathrm{C} \mathrm{NMR}(68.7 \mathrm{MHz})$ of the methine group of PVA in aqueous solutions of PVA and iron(III) ${ }^{\mathbf{a}}$

\begin{tabular}{cccc}
\hline & \multicolumn{3}{c}{$1 / T_{1 \mathrm{p}} / \mathrm{s}^{-1}$} \\
\cline { 2 - 4 } & $m m^{\mathrm{b}}$ & $\mathrm{rm}^{\mathrm{c}}$ & $r r^{\mathrm{d}}$ \\
\hline 4.0 & 1.04 & 1.22 & 1.00 \\
7.0 & 0.91 & 1.13 & 0.79 \\
\hline
\end{tabular}

${ }^{\mathrm{a}}[\mathrm{PVA}]=400 \mathrm{~mm} ; R=40 ; \mathrm{PVA}, \mathrm{DP}=33 ; \mathrm{DS}=95 ; 25 \pm$ $0.1^{\circ} \mathrm{C}$. ${ }^{\mathrm{b}}$ Isotactic triad. ${ }^{\mathrm{c}}$ Heterotactic triad. ${ }^{\mathrm{d}}$ Syndiotactic triad.

mode of hydrogen bonds between two neighboring PVA chains or remote hydroxyl groups of a PVA chain. Consequently, Table IV also implies that such a hydrogen bonding does not seem to affect the complex structures and, furthermore, that the hydroxyl groups of PVA do never coordinate directly to the iron(III) ions. After all, it is probable that these hydroxyl groups in the complexes are oriented toward the outside bulk water with some structural flexibility. More detailed information on tacticity effects on the complexation will be obtained by experiments with isotactic and syndiotactic PVAs. However, we must leave this for a future study.

\section{Molecular Motion of the Iron(III) Com- plexes with $P V A$}

The $\mathrm{pD}$ dependence of $1 / T_{1 \mathrm{p}}$ for PVA protons in aqueous solutions of the complexes over the pD range 3 to 9 was measured with two NMR spectrometers working at different frequencies. From the ratio of two $1 / T_{1 p}$ values obtained for each of the protons, the correlation time for dipolar interaction, $\tau_{\mathrm{c}}$, was determined by using eq 2, as shown in Figure 5. $\tau_{\mathrm{c}}$ is regarded as a measure of molecular mobility in solution and influenced by many factors, such as molecular size, solution viscosity, and temperature. ${ }^{22} \tau_{\mathrm{c}}$ for paramagnetic metal complexes is given by

$$
\frac{1}{\tau_{\mathrm{c}}}=\frac{1}{\tau_{r}}+\frac{1}{\tau_{s}}+\frac{1}{\tau_{m}}
$$

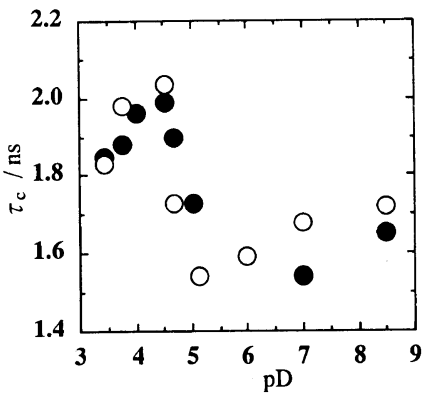

Figure 5. $\mathrm{pD}$ dependence of $\tau_{\mathrm{c}}$ for the aqueous solutions of PVA and iron(III) $(\mathrm{DP}=250, \mathrm{DS}=98 ;[\mathrm{PVA}]=400 \mathrm{mM}$; $\left.R=40 ; 25 \pm 0.1^{\circ} \mathrm{C}\right): 0, \mathrm{CH}_{2} ; \mathrm{O}, \mathrm{CH}$.

where $\tau_{\mathrm{r}}$ is the rotational correlation time, $\tau_{\mathrm{s}}$ is the electron-spin relaxation time, and $\tau_{\mathrm{m}}$ is the correlation time for ligand exchange. Figure 5 shows a decrease of $\tau_{c}$ with a rise in $\mathrm{pD}$ from 4. $\tau_{\mathrm{s}}$ and $\tau_{\mathrm{m}}$ are probably constant above $\mathrm{pD} 4$, since the iron(III) clusters formed in the same $\mathrm{pD}$ range remain unchanged in structure, as has already been described above. Therefore, the above-mentioned descent of $\tau_{c}$ is attributed to that of $\tau_{\mathrm{r}}$. This means a decrease in molecular size or a molecular contraction above pD 4 , and is consistent with the fact that aqueous solutions of iron(III) and the PVA of DP $<500$ remarkably decrease in viscosity above $\mathrm{pH} 3 .^{27}$

Figure 5 also shows that $\tau_{\mathrm{c}}$ for the $\mathrm{CH}_{2}$ protons is comparable to that for the $\mathrm{CH}$ ones over the whole $\mathrm{pD}$ range, suggesting that the complex molecules are in isotropic motion in solution, as if they are spherical in shape.

\section{CONCLUSION}

In this study, both iron(III) and copper(II) complexes with many PVAs of different DP values have been investigated by NMR techniques in order primarily to obtain more detailed information on their structures, with stronger emphasis on the former complexes, since we have already reported some aspects of the latter. ${ }^{9}$ The complex formation between PVA and iron(III) is substantially the same as that between PVA and copper(II), in respect 

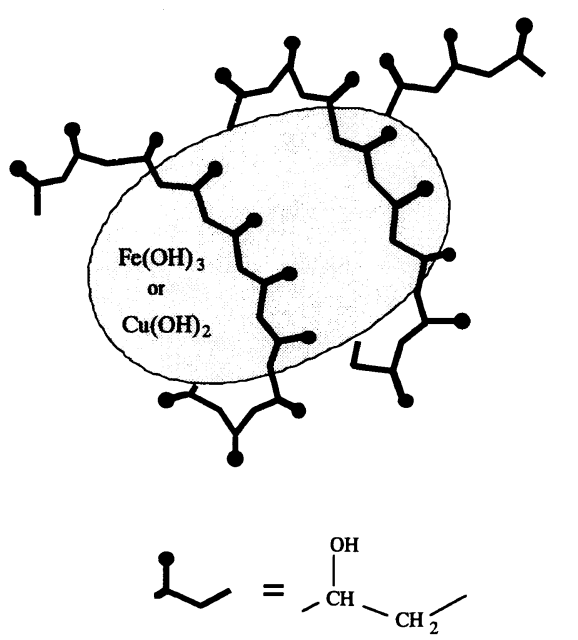

Figure 6. A schematic representation for the structure of iron(III) and copper(III) complexes with PVA.

of the inclusion of metal hydroxide-like clusters by PVA through hydrophobic interaction. This fact suggests that this type of complex formation is general and important in aqueous solutions of metal ions and water-soluble polymers, together with the idea that similar kinds of metal complexes with polyols may be encountered everywhere in biological and natural environmental systems. A schematic representation for the structure of the complexes of ion(III) and copper(III) with PVA is tentatively given in Figure 6.

In preparing the water-soluble metal complexes with PVA, it is necessary to add PVA to an aqueous solution of the metal ions, not after the hydrolysis and precipitation of them, but before that. During the hydrolysis, PVA probably gives a reaction environment and controls the formation and structure of the metal hydroxide-like clusters, simultaneously forming hydrophobic cages or pockets for the inclusion of the clusters. This complex formation is of a new type after all. However, much effort is necessary to obtain further detailed structural information on the complexes. There are still many simple but fundamental questions not answered yet, for example, whether any one of the $\mathrm{OH}$ groups of PVA never interacts directly with metal hydroxide-like clusters and why the complexes of copper(II) with PVA are green. ${ }^{9}$ Solutions for these problems are the subject for a future study.

\section{REFERENCES}

1. F. M. Herman and G. G. Norman, Ed., "Encyclopedia of Polymer Science and Technology," Vol. 14, Wiley-Interscience, New York, N. Y., 1977, p 149.

2. E. Ahad, J. Appl. Polym. Sci., 18, 1587 (1974).

3. E. Perzron, L. Liebler, A. Ricard, F. Laufuma, and R. Audebert, Macromolecules, 22, 1169 (1989).

4. H. Takayama, T. Matsuyama, K. Tamura, and S. Matsuzawa, Rept. Progr. Polym. Phys. Jpn., 33, 225 (1990).

5. C. Dittmar and W. J. Priest, J. Polym. Sci., 18, 275 (1955).

6. H. Yokoi, K. Yagishita, and Y. Nakanishi, Bull. Chem. Soc. Jpn., 63, 746 (1990).

7. (a) R. Rossetti, J. L. Ellison, J. M. Gibson, and L. E. Brus, J. Chem. Phys., 80, 4464 (1984); (b) Y. Wang, H. Liu, and Y. Jiang, J. Chem. Soc., Chem. Commun., 1878 (1989); (c) C.-C. Yen, T.-C Chang, and H. Kakinoki, J. Appl. Polym. Sci., 63, 746 (1990); (d) T. Yamamoto, "Kobunshi Sakutai (Dynamic Interactions and Electronic Processes of Macromolecular Complexes)," E. Tsuchida, Ed., Japan Scientific Society Press, Tokyo, 1991, p 333; (e) H. Hirai, H. Wakabayashi, and M. Komiyama, Bull. Chem. Soc. Jpn., 59, 545 (1986); (f) S. Kitano, Y. Koyama, K. Kataoka, T. Okano, and Y. Sakurai, J. Controlled Release, 19, 162 (1992); (g) M. Tanaka, H. Yamazaki, and H. Kakinoki, Kobunshi Ronbunshu, 51, 447 (1994).

8. H. Yokoi, "Kobunshi Sakutai (Dynamic Interactions and Electronic Processes of Macromolecular Complexes)," E. Tsuchida, Ed., Japan Scientific Society Press, Tokyo, 1991, p 181.

9. H. Yokoi, S. Kawata, and M. Iwaizumi, J. Am. Chem. Soc., 108, 3358 (1986).

10. H. Yokoi, Y. Mori, K. Yagishita, and Y. Fujise, J. Mater. Chem., to be published.

11. (a) H. J. Kuhn and I. Z. Toth, Naturforsh, 18A, 112 (1963); (b) N. Hojo, H. Shirai, and S. Hayashi, J. Polym. Sci., C, 47, 299 (1974).

12. M. Sumi, J. Suenaga, H. Tamaki, I. Komamine, and M. Asakura, Kagakusenni Kenkyu Kaishi, 18, 7 (1979).

13.. S. Matsumoto and Y. Ohyanagi, Kobunnshi Kagaku, 17, 1 (1960).

14. P. K. Glasoe and F. A. Long, J. Phys. Chem., 64, 188 (1960).

15. G. C. Levy and I. R. Peat, J. Magn. Reson., 18, 
500 (1975).

16. S. Meiboon and D. Gill, Rev. Sci. Instrum., 1934 (1985).

17. (a) I. Solomon, Phys. Rev., 99, 559 (1955); (b) N. Bloembergen, J. Chem. Phys., 27, 572 (1957).

18. N. Bloembergen and L. O. Morgan, J. Chem. Phys., 34, 842 (1961).

19. T. Iida, T. Iizuka, and I. Morishima, "NMR no Seikagakueno Ouyo," Kagakuzokan 67 (in Japanese), Kagakudojin, Tokyo, 1976, p 99.

20. H. Yokoi, Y. Mori, T. Mitani, and S. Kawata, Bull. Chem. Soc. Jpn., 65, 1989 (1992).

21. (a) S. Mann, J. Chem. Soc., Dalton Trans., 1 (1993); (b) L. Addadi and S. Weiner, Angew. Chem. Int. Ed. Engl., 31, 153 (1992); (c) R. J. Davy, S. N. Black, L.
A. Bromley, D. Cottier, B. Dobbs, and J. E. Rout, Nature, 353, 549 (1991).

22. A. F. Wells, "Structural Inorganic Chemistry," Clarendon Press, Oxford, 1984, p 633.

23. I. Bertini and C. Luchinat, "NMR of Paramagnetic Molecules in Biological Systems," A. B. P. Lever and H. B. Gray, Ed., The Benjamin/Cummings Publishing Company Inc., California, 1986, p 100.

24. K. Tsuchiya, K. Takegoshi, and K. Hikichi, Polym. J., 26, 479 (1994).

25. W. O. Deric, Macromolecules, 17, 1458 (1984).

26. A. Takayama and S. Hiramitsu, Polym. J., 6, 103 (1974).

27. H. Yokoi, Y. Kenmochi, and Y. Mori, Macromolecules, to be published. 